\title{
Screening of Capillaria philippinensis infection using Trichuris muris and Trichinella spiralis antigens
}

\author{
Original Mona I Ali ${ }^{1}$, Nadia A El-Dib ${ }^{2}$, Mahmoud Abdel-Latif ${ }^{3}$, Waleed M Arafa $^{4}$, Samah S \\ Article Abdel Gawad ${ }^{1}$
}

Departments of Medical Parasitology Faculty of Medicine, Beni-Suef ${ }^{1}$ and Cairo ${ }^{2}$ Universities, Immunity Division, Department of Zoology, Faculty of Science ${ }^{3}$ and Medical Parasitology, Faculty of Veterinary Medicine ${ }^{4}$, Beni-Suef University, Egypt

\begin{abstract}
Background: Intestinal capillariasis is a disease caused by Capillaria philippinensis. Human infections became more prevalent in many countries including Egypt. This nematode is related to Trichinella and Trichuris species, all of them belong to Trichinelloidea superfamily. Diagnosis of intestinal capillariasis may be missed by stool examination.

Objective: This study aimed to use enzyme-linked immunosorbent assay (ELISA) and immunoblotting in screening for intestinal capillariasis as a practical and rapid diagnostic test.

Subjects and Methods: Trichuris muris and Trichinella spiralis adult worms were isolated from infected mice and crude antigens were prepared. The protein content for both adult worm extracts was determined. Human blood samples were collected from 20 capillariasis patients, 20 control individuals and $10 W$. bancrofti infected patients. The study evaluated cross-reactivity between T. spiralis and T. muris antigens and sera from cases infected with $C$. philippinensis using ELISA and immunoblotting.

Results: In ELISA, T. muris crude antigen cross reacted with $100 \%$ of capillariasis sera with $100 \%$ sensitivity and specificity and cross reacted with $10 \%$ of sera from bancroftian filariasis. T. spiralis crude antigen cross reacted with $50 \%$ of capillariasis sera, and $9 \%$ of sera from bancroftian filariasis. Neither $T$. muris nor T. spiralis crude antigens reacted with sera from control group. Immunoblotting results showed that IgG antibodies from control group didn't recognize specific proteins in T. muris antigen, while in $W$. bancrofti group, only one band in one sample appeared (100-135 kDa). IgG antibodies from capillariasis cases recognized multiple common protein bands (35-180 kDa). IgG antibodies from capillariasis, control and $W$. bancrofti sera did not recognize specific proteins in T. spiralis crude antigen.

Conclusion: Antigens from T. muris and T. spiralis can be used successfully to detect infection with $C$. philippinensis using serum samples of cases by ELISA. Crude antigen of T. muris gave better results than that of T. spiralis. Immunoblotting can be used for diagnosis of capillariasis by using crude antigen of $T$. muris.
\end{abstract}

Keywords: ELISA, immunodiagnosis, intestinal capillariasis, Trichinelloidea, western blotting.

Received: 21 May, 2021, Accepted: 10 August, 2021.

Corresponding Author: Mona I. Ali, Tel.: +20 1221969196, E-mail: monaib.para@yahoo.com

Print ISSN: 1687-7942, Online ISSN: 2090-2646, Vol. 14, No. 2, August, 2021.

\section{INTRODUCTION}

The genus Capillaria is a member of the superfamily Trichinelloidea. From this group only three major genera: Trichuris, Trichinella and Capillaria cause human diseases. All Trichinnelloidae members have a unique structure of the esophagus with the presence of stichocytes that differentiates them from other nematodes ${ }^{[1]}$. C. philippinensis is a nematode of fisheating birds that causes intestinal capillariasis in humans. It first appeared in the Philippines where the disease seems to be endemic ${ }^{[2]}$. In Thailand, a large number of cases were identified ${ }^{[3]}$. Subsequent cases were diagnosed in many parts of the world including Korea ${ }^{[4]}$, Taiwan, and China ${ }^{[5,6]}$. Youssef et $a l .{ }^{[7]}$ reported the first case in Egypt after which sporadic cases were diagnosed particularly in Upper
Egypt ${ }^{[8-13]}$. Egypt showed the highest number of cases detected outside the endemic areas in the Philippines and Thailand ${ }^{[10]}$.

Infection with $C$. philippinensis is mainly acquired through ingestion of the parasite larva in raw, undercooked, small fresh water or brackish water fish or by contaminated fingers during fish evisceration ${ }^{[10]}$. Infection leads to inflammation and atrophy of the intestinal villi with subsequent malabsorption of nutrients resulting in extreme protein-deficient enteropathy ${ }^{[14,15]}$. Patients with intestinal capillariasis usually suffer from chronic diarrhea ${ }^{[16]}$. The disease can be fatal if diagnosis and treatment are delayed. In non-endemic areas, diagnosis is often skipped due to lack of knowledge of the nature of this disease $\mathrm{e}^{[17]}$. Some cases are not easily diagnosed and had to pass 
through many sophisticated, expensive, and sometimes invasive techniques to be diagnosed ${ }^{[10]}$.

This study is based on the concept of similarity between members of the Trichinelloidea superfamily, and the possible cross reactivity between patient sera and antigens from members of the superfamily, which can add a new diagnostic method for the detection of the disease. The aim of the study is to establish a rapid, easy, and accurate diagnostic tool for infection with C. philippinensis, as the direct methods are not always positive ${ }^{[18]}$.

\section{SUBJECTS AND METHODS}

This descriptive analytical study was conducted at Medical Parasitology Department, Faculty of Medicine, and Department of Zoology, Immunity Division, Faculty of Science, Beni-Suef University from 2018 to 2020.

Preparation of adult worm somatic extracts: T. muris adult worms were isolated from laboratory infected mice and the homogenate preparation was carried out as previously described ${ }^{[19,20]}$. Briefly, worms were washed thoroughly in PBS, homogenized in $50 \mathrm{mM}$ Tris $\mathrm{HCl}$ ( $\mathrm{pH}$ 8.8) containing $0.15 \mathrm{M} \mathrm{NaCl}, 1 \mathrm{mM}$ EDTA, $1 \mathrm{mM}$ 1,4-Dithiothreitol and $50 \mathrm{mM}$ Phenylmethylsulfonyl fluoride using motor driven homogenizer (REMI, Maharashtra, India) at $4^{\circ} \mathrm{C}$. The extract was centrifuged at $5000 \times \mathrm{g}$ followed by $15,000 \times \mathrm{g}$ for $30 \mathrm{~min}$ and the clear supernatant was separated.

For T. spiralis antigen, adult worms were collected from the small intestine of experimentally infected Wistar rats at 4 days after experimental infection. The collected adult worms were washed several times in PBS and then homogenized in lysis buffer containing 7 M urea, 2 M thiourea, 4\% 3-(3-Cholamidopropyl) dimethylammonio-1-propanesulfonate hydrate $65 \mathrm{mM}$ Tris, 2\% DTT, and 1\% Bio-Lyte (pH 3-10). The adult worms lysates were centrifuged at $20,000 \times \mathrm{g}$ at $4^{\circ} \mathrm{C}$ for $60 \mathrm{~min}$ and the supernatant was collected and used as adult extract ${ }^{[21]}$. The protein content for both adult worm extracts was determined by Bicinchoninic acid assay kit (Sigma) ${ }^{[22]}$.

Human sera: Human blood samples were collected from 20 patients who had been diagnosed by stool samples analysis as having $C$. philippinensis infection by detecting eggs, adults and/or larvae in stool; from 20 control individuals who had not experienced any parasitic diseases; and 10 samples were from patients with $W$. bancrofti infections. Stool examination of the last two groups showed no other nematodes eggs such as A. lumbricoides, A. duodenale, and T. trichuria.

ELISA: The optional dilutions of various reagents were determined using checkerboard titration. The assay was performed as previously described ${ }^{[23]}$. Briefly, 96-well ELISA plates (Corning, USA) were coated with adult worm antigens $(2.0 \mu \mathrm{g} / \mathrm{ml})$ in $100 \mu \mathrm{l}$ of bicarbonate buffer ( $\mathrm{pH} 9.6)$ overnight at $4^{\circ} \mathrm{C}$. After blocking with PBS $0.1 \%$ Tween 20 (PBST) containing $5 \%$ fetal calf serum at room temperature for $2 \mathrm{~h}$, the following reagents were sequentially added and incubated at $37^{\circ} \mathrm{C}$ for $1 \mathrm{~h}$ : (1) human sera diluted $1: 100$ in PBST, and (2) horseradish peroxidase-labeled goat anti-human (HRP) IgG antibody (Sigma, USA) diluted 1:5000. The reactions were detected by the addition of the substrate o-phenylenediamine dihydrochloride (OPD; Sigma, USA) plus H2O2 and stopped with $50 \mu \mathrm{l} /$ well of 2 M H2SO4. Optical density (OD) values at 490 $\mathrm{nm}$ were measured with a microplate reader (TECAN, Austria). All samples were run in duplicate. The cut-off value of the ELISA was evaluated for the two antigen preparations based on a calculation of mean control OD $+(2 \times \mathrm{SD})$. The recorded ODs above the cut-off value were regarded as positive.

Western blotting: Immunoblotting was carried out as previously described ${ }^{[24]}$. Adult worm extracts were separated in SDS-10\% polyacrylamide gels and transferred to nitrocellulose membranes $(0.45 \mu \mathrm{m}$; Heidelberg; Serva Electrophoresis GmbH, Germany) by electroblotting. Membranes were washed in PBS/ Tween buffer (PBS containing 0.3\% Tween-20) and incubated for $1 \mathrm{~h}$ at room temperature in blocking buffer containing 5\% non-fat milk in PBS/Tween-20, followed by washing and incubation with the human serum (1:100) in washing buffer overnight at $4^{\circ} \mathrm{C}$. Formed immunocomplexes were detected by HRP anti-human IgG antibody (1:5000; KPL, Maryland, USA). After $2 \mathrm{~h}$ of incubation at room temperature, bands were developed by adding substrate $(50 \mathrm{mg}$ 3,3'-Diaminobenzidine tetrahydrochloride and $100 \mu \mathrm{l}$ $\mathrm{H} 202$ in $100 \mathrm{ml} \mathrm{PBS}$ ).

Statistical analysis: SPSS (version 20) statistical program (SPSS Inc., Chicago, IL) was used to carry out one-way analysis of variance (ANOVA) on our data. When significant differences by ANOVA were detected, analysis of differences between the means of the OD values was performed by Dunnett's t-test. The sensitivity and specificity of ELISA were assessed according to the following formulas: Sensitivity $=$ no. of true positives/(no. of true positives + no. of false negatives $) \times 100$; and specificity $=$ no. of true negatives $/$ (no. of false positives + no. of true negatives) $\times 100^{[25]}$. Positive and negative predictive values and diagnostic accuracy were also calculated. $P$ values $<0.5$ were considered significant.

Ethical approval: The study was approved by the Research Ethical Committee of the Beni-Suef University, Faculty of Medicine, Egypt. Signed informed consent was obtained from all individuals included in the study after explaining the purpose of the study. 


\section{RESULTS}

Detection of cross-reactive human IgG using ELISA: When T. muris crude antigen was tested for serodiagnostic cross-reacting human IgG, sero-positive reactions were found in $100 \%$ from capillariasis and $10 \%$ from bancroftian filariasis cases. Calculated sensitivity and specificity for capillariasis were both $100 \%$ (Table 1). The difference between the means of OD for control and Capillaria or Capillaria and $W$. bancrofti were statistically significant $(P<0.01$ and $<0.001$, respectively) (Fig. 1). In addition, Trichinella crude antigen tested for serodiagnostic cross-reacting human IgG recorded positive reactions in 50\% from capillariasis and 9\% from bancroftian filariasis (Fig. 2). The mean of OD for the reaction with Capillaria was significantly higher than reaction with control and $W$. bancrofti infections $(P<0.05)$ (Fig. 2). Calculated sensitivity and specificity were $50 \%$ and $100 \%$ respectively for $T$. spiralis (Table 1 ).

Detection of specifically recognized proteins in adult worm antigens using immunoblotting: IgG antibodies from selected controls $(n=20)$ did not
Table 1. Diagnostic yield and accuracy of ELISA results using T. muris Ag and T. spiralis Ag among capillariais patients.

\begin{tabular}{llcc}
\hline \hline & & T. muris Ag & T. spiralis Ag \\
\hline \multirow{6}{*}{ ELISA } & Sensitivity & $100 \%$ & $50 \%$ \\
& Specificity & $100 \%$ & $100 \%$ \\
& PPV & $100 \%$ & $100 \%$ \\
& NPV & $100 \%$ & $66.7 \%$ \\
& Accuracy & $100 \%$ & $75 \%$ \\
\hline \hline
\end{tabular}

recognize specific proteins in T. muris crude antigen. In contrast, IgG antibodies from capillariasis cases $(n=20)$ could recognize multiple common protein bands ranging between $35-180 \mathrm{kDa}(35,48,63,75$, $100,135,180 \mathrm{kDa})$. IgG antibodies from $W$. bancrofti antisera $(n=10)$ did not recognize specific proteins in T. muris crude antigen except in only one serum sample (No. 8), that recognized a protein band (100-135 kDa) (Fig. 3). IgG antibodies from capillariasis, control and other $W$. bancrofti infections did not recognize specific proteins in T. spiralis crude antigen.

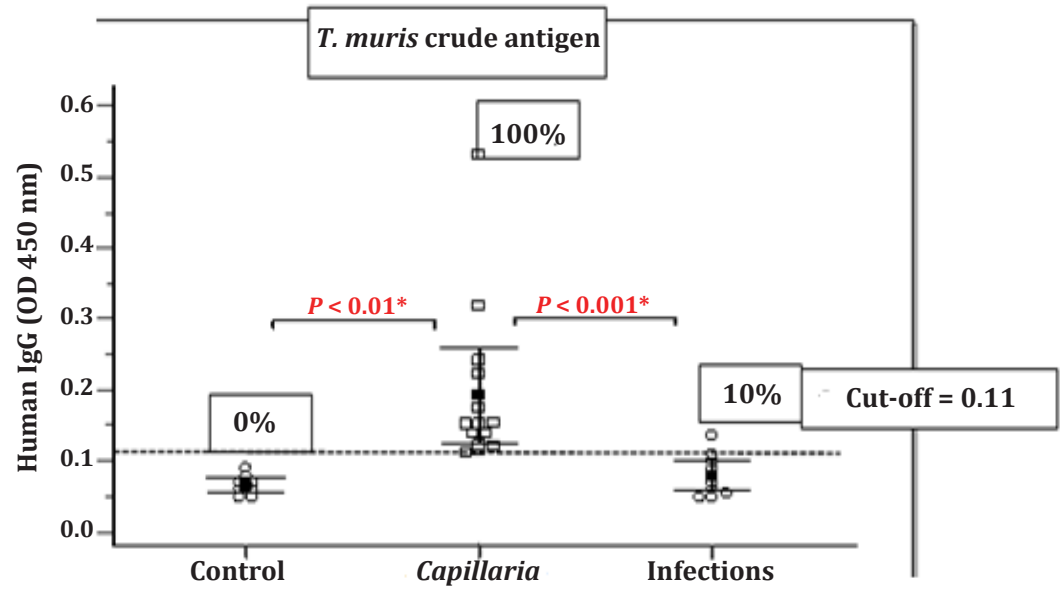

Fig. 1. T. muris crude antigen cross reacted with $100 \%$ of capillariasis sera, and $10 \%$ of other parasitic infections (bancroftian filariasis); *: Sifnificant $(P<0.05)$.

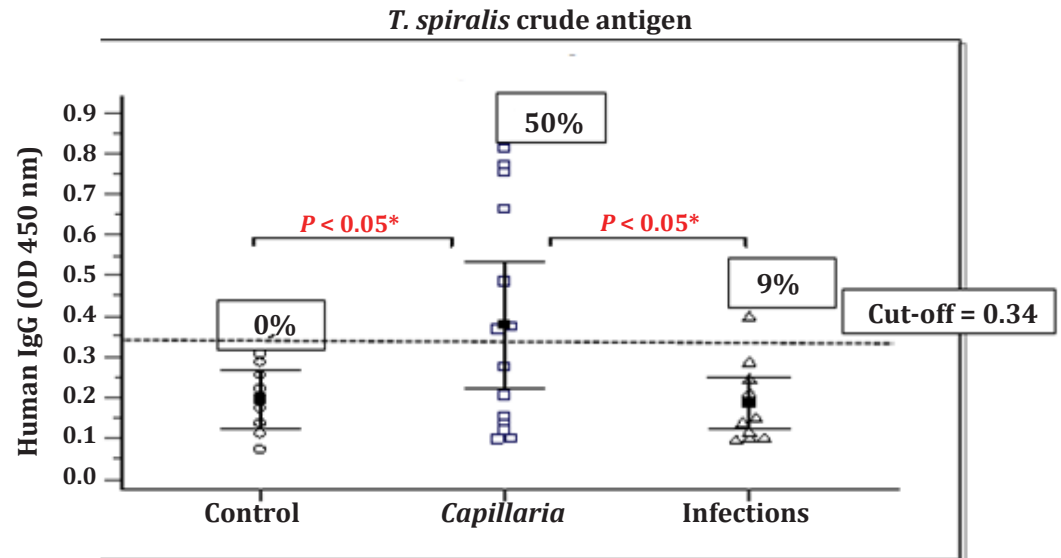

Fig. 2. T. spiralis crude antigen cross reacted with $50 \%$ of capillariasis and $9 \%$ of other parasitic infections (bancroftian filariasis); *: Sifnificant $(P<0.05)$.. 

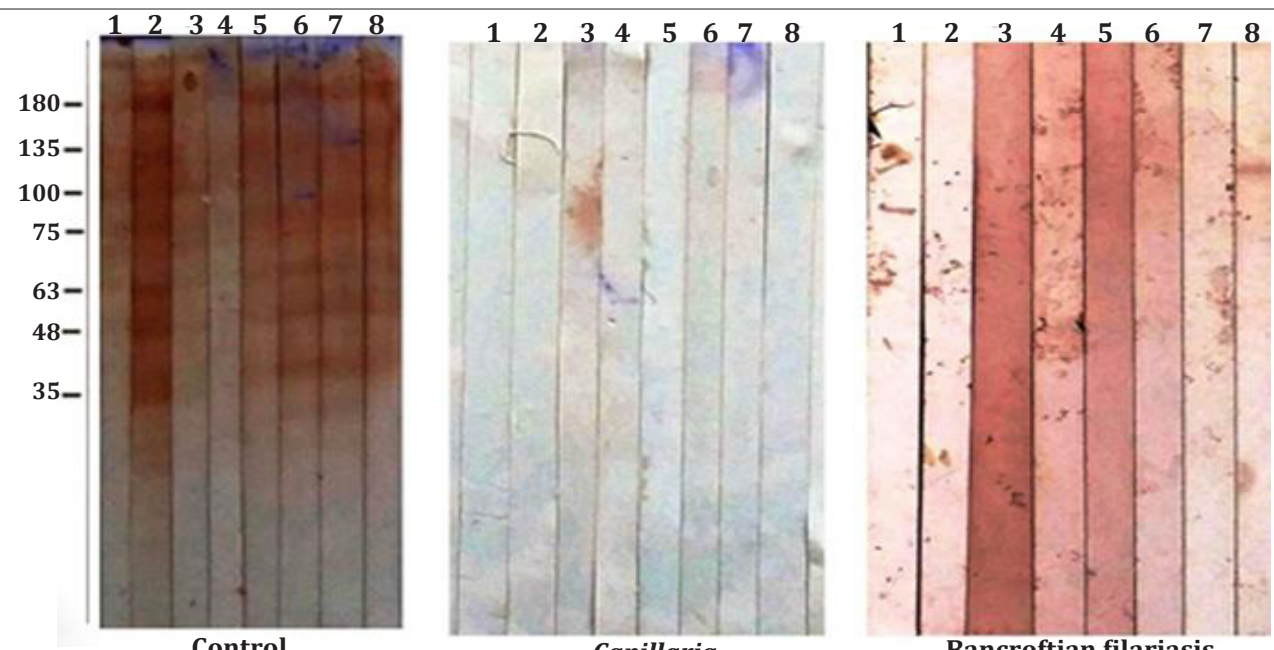

Fig. 3. Western blot. Sera from control did not recognize specific proteins in T. muris crude antigen. IgG antibodies from Capillaria positive cases could recognize multiple common protein bands ranging between $35-180 \mathrm{kDa}$. There is an apparent band (100-135 $\mathrm{kDa}$ ) in serum no. (8) in bancroftian filariasis group.

\section{DISCUSSION}

The real prevalence of $C$. philippinensis may be higher than predicted, but the lack of awareness of the parasite and the outcome of infection by physicians and laboratory technicians is delaying the diagnosis ${ }^{[3]}$. Immunodiagnosis may allow early detection of the parasite. Using it as an extra diagnostic tool helps to detect $C$. philippinensis infection ${ }^{[18]}$. In our study, the reactivity of serum samples from 20 cases diagnosed as human $C$. philippinensis infection was examined by ELISA and immunoblotting against $T$. muris and T. spiralis antigens. By ELISA, 100\% of the C. philippinensis cases were reactive with $T$. muris antigen and $50 \%$ of cases reacted with $T$. spiralis antigens with statistical significance. Only $10 \%$ of bancroftian filariasis antisera reacted with T. muris antigen while $9 \%$ reacted with $T$. spiralis antigens, indicating the superiority of the former as a diagnostic antigen. The similarity of stichocytes between the members of the family Trichinelloidae, allows for the use of their antigens in immunological reactions ${ }^{[26]}$.

These cells are rich in antigens, that can be used for the serodiagnosis of capillariasis ${ }^{[27]}$. Similar studies consistent with our results were conducted worldwide. Intapan et al., ${ }^{[27]}$ diagnosed intestinal capillariasis using $T$. spiralis antigen by ELISA that reacted positively with $75 \%$ of trichinellosis. Another study conducted by Banzon et al. ${ }^{[28]}$ proved that $C$. philippinensis sera were also reactive with $T$. spiralis and T. vulpis antigens.

To avoid the false negative diagnosis of infection with $C$. philippinensis in the absence of eggs in stool, an immunological study was carried by El-Dib et al. ${ }^{[18]}$ in which coproantigen was extracted from the stools of infected patients. Hyperimmune serum was prepared against this antigen and used successfully to detect the copro antigen of $C$. philippinensis in stool samples of suspected cases by sandwich ELISA.
By Immunoblotting, IgG antibodies from capillariasis cases could recognize multiple common protein bands in $T$. muris crude antigen ranging between $35-180 \mathrm{kDa}(35,48,63,75,100,135,180$ $\mathrm{kDa}$ ). No bands were detected in the control group. Similarly, Abdel-Rahman et al. ${ }^{[29]}$ used C. philippinensis coproantigen and egg antigen for diagnosis of intestinal capillariasis utilizing the immunoblotting method. Results showed no bands in the control group, while multiple protein bands were recognized for both antigens in capillariasis cases. These bands ranged from 10 to $148 \mathrm{KDa}$ for C. philippinensis coproantigen and from 60 to 62 for egg antigen. On the other hand, in our study IgG antibodies from $W$. bancrofti antisera did not recognize specific proteins in T. muris crude antigen except in only serum sample that recognized a single protein band (100-135 $\mathrm{kDa}$ ). There may be cross reactivity between filarial infections and other nematodes including Capillaria as tested by Joekel et al. ${ }^{[30]}$ who proved immunological cross-reactions between Dirofilaria and Capillaria aerophila in expermintally infected dogs.

In conclusion, this study showed that antigen from T. muris and T. spiralis can be used successfully to detect infection with $C$. philippinensis using serum samples of cases by ELISA technique. Crude antigen of T. muris gave better results than crude antigens of T. spiralis. Western blot technique also can be used for diagnosis of capillariasis by utilizing crude antigen of T. muris.

Author contributions: All authors contributed to the study conception and design, the practical work, results interpretation, statistical analysis and writing the manuscript.

Conflict of interest: All authors declare that they have no conflict of interest. Funding: No funding. 


\section{REFERENCES}

1. Mc Carthy J, Moore TA. Emerging helminth zoonoses. Int J Parasitol 2000; 30: 1351-1360.

2. Cross JH. Intestinal capillariasis. Clin Microbiol Rev 1992; 5: 120-129.

3. Saichua P, Nithikathkul C, Kaewpitoon N. Human intestinal capillariasis in Thailand. World J Gastroenterol 2008; 14:506-510.

4. Lee SH, Hong ST, Chai JY, Kim WH, Kim YT, Song IS, et al. A case of intestinal capillariasis in the Republic of Korea. Am J Trop Med Hyg 1993; 48: 542-546.

5. Hwang KP. Human intestinal capillariasis (Capillaria philippinensis) in Taiwan. Zhonghua Minguo Xiao Er Ke Yi Xue Hui Za Zhi 1999; 39: 82-85.

6. Bair MJ, Hwang KP, Wang TE, Liou TC, Lin SC, Kao CR et al. Clinical features of human intestinal capillariasis in Taiwan. World J Gastroenterol 2004; 10: 2391-2393.

7. Youssef FG, Mikhail EM, Mansour NS. Intestinal capillariasis in Egypt: a case report. Am J Trop Med Hyg 1989; 45:195-196.

8. El-Dib NA, Ahmed JA, El-Arousy M, Mahmoud MA, Garo $\mathrm{K}$ : Parasitological aspects of Capillaria philippinensis recovered from Egyptian patients. J Egypt Soc Parasitol 1999; 29:139-147.

9. Ahmed L, El-Dib NA, El-Boraey Y, Ibrahim M. Capillaria philippinensis: an emerging parasite causing severe diarrhea in Egypt. J Egypt Soc Parasitol 1999; 29: 483493.

10. El-Dib NA, Doss WH. Intestinal capillariasis in Egypt; epidemiological background. J Egypt Soc Parasitol 2002; 32: 145-154.

11. El-Karaksy H, El-Shabrawi M, Mohsen N, Kotb M, El-Koofy N, El- Deeb N. Capillaria philippinensis: a cause of fatal diarrhea in one of two infected Egyptian sisters. J Trop Pediatr 2004; 50: 57-60.

12. Attia RAH, Tolba MEM, Younes DA, Bakir H, El-Deek HEM, Kamel S. Capillaria philippinensis in Upper Egypt: Has it become endemic? Am J Trop Med Hyg 2012; 86(1): 126133.

13. Ali MI, El-Badry AA, Rubio JM, Ghieth MA, El-Dib NA. Prevalence of Capillaria philippinensis in diarrheic patients using the small subunit ribosomal DNA (ssurDNA) gene. Sci Parasitol 2016; 17(3-4): 93-100.

14. Cross JH. Capillariasis. in: Palmer SR, Soulsby L, Simpson DIH, eds. Zoonoses: Biology, Clinical Practice, and Public Health Control. New York, Oxford University Press 1998; 759-772.

15. Sangchan A, Wongsaensook A, Kularbkaew C, Sawanyawisuth K, Sukeepaisamgaroen W, Mairiang P. The endoscopic pathologic finding in intestinal capillariasis: case report. J Med Assoc Thai 2007; 90: 175-178.

16. Limsrivilai J, Pongprasobchai S, Apisarnthanarak P, Manatsathit S. Intestinal capillariasis in the $21^{\text {st }}$ century: clinical presentations and role of endoscopy and imaging. BMC Gastroenterol. 2014; 14:207.
17. Vasantha PL, Girish N, Sai Leela K. Human intestinal capillariasis. A rare case report from non-endemic area (Andhra Pradesh, India). Indian J Med Microbiol 2012; 30(2): 236-239.

18. El-Dib NA, Sabry MA, Ahmed JA, El-Basiouni SO, El-Badry AA. Evaluation of Capillaria philippinensis coproantigen in the diagnosis of infection. J Egypt Soc Parasitol 2004; 34(1): 97-106.

19. Cooper PJ, Chico ME, Vaca MG. Risk factors for asthma and allergy associated with urban migration: background and methodology of a cross-sectional study in AfroEcuadorian school children in Northeastern Ecuador (Esmeraldas-SCAALA Study). BMC Pulm Med 2006; 6: 24.

20. Abdel-Latif M, Sakran T. Detection for cross-reactive proteins in filarial worm Setaria equina, MCF-7 human breast cancer, and Huh-7 hepatoma cells. J Immunoassay Immunochem 2016; 37(6): 572-84.

21. Yang J, Pan W, Sun X, Zhao X, Yuan G, Sun Q, et al. Immunoproteomic profile of Trichinella spiralis adult worm proteins recognized early infection sera. Parasit Vectors 2015; 8:20.

22. Smith PK, Krohn RI, Hermanson GT, Mallia AK, Gartner FH, Provenzano MD et al. Measurement of protein using bicinchoninic acid. Anal Biochem 1985; 150 (1): 76-85.

23. Bahgat MM, Saad AH, El-Shahawi GA, Gad AM, Ramzy RM, Ruppel A, et al. Cross-reaction of antigen preparations from adult and larval stages of the parasite Setaria equine with sera from infected humans with Wuchereria bancrofti. East Mediterr Health J 2011; 17(8): 679-86.

24. Abdel-Latif M, Sakran T, Badawi YK, Abdel-Hady DS. Influence of Moringa oleifera extract, vitamin $\mathrm{C}$, and sodium bicarbonate on heat stress induced HSP70 expression and cellular immune response in rabbits, Cell Stress Chaperones 2018; 23: 975-984.

25. Zhong ZR, Zhou HB, Li XY, Luo QL, Song XR, Wang W, et al. Serological proteome-oriented screening and application of antigens for the diagnosis of schistosomiasis japonica. Acta Trop. 2010; 116(1): 1-8.

26. Anderson RC. Nematode parasite of vertebrates, their development and transmission. Wallingford. Oxon: $\mathrm{CAB}$ International 1992; 544 - 50.

27. Intapan PM, Maleewong W, Sukeepaisarnjaroen W, Morakote N. Potential use of Trichinella spiralis antigen for serodiagnosis of human capillariasis philippinensis by immunoblot analysis. Parasitol Res 2005; 98 (3): 227-31.

28. Banzon TC, Lewert RM, Yogore, MG. Serology of Capillaria philippinensis infection: reactivity of human sera to antigen prepared from Capillaria obsignata and other helminths. Am J Trop Med Hyg 1975; 24 (2): 256-263.

29. Abdel-Rahman SM, Moneib ME, Sha-hin MS, Abdel Aziz LA. Immuno-diagnosis of Capillaria philippinensis by western blot using coproantigen and egg antigen. El-Mini Med Bull 2005; 16, 2: 9-17.

30. Joekel DE, Maier S, Huggel K, Schaper R, Deplazes P. Specific antibody detection in dogs with filarial Infections. Parasitol Res 2017; 116 (Suppl 1):81-90. 